\title{
Vector Critical Points and Cone Efficiency in Nonsmooth Vector Optimization
}

\author{
Tadeusz Antczak* and Marcin Studniarski
}

\begin{abstract}
In this paper, a nonsmooth vector optimization problem with cone and equality constraints is considered. We establish some relations between the notions of vector critical points in the sense of Fritz John and in the sense of Karush-Kuhn-Tucker and weakly $K$-efficient and $K$-efficient solutions for the constrained vector optimization problem in which every component of the involved functions is locally Lipschitz. These relationships are stated under cone- $F J$-pseudo-invexity and cone- $K T$-pseudoinvexity hypotheses defined for the considered vector optimization problem with cone inequality and also equality constraints and via the Clarke generalized gradient for vector-valued functions.
\end{abstract}

\section{Introduction}

Optimization problems, in which decisions are made taking into account several conflicting criteria, rather than by optimizing a single objective, are called multiobjective programming or vector optimization. An optimal solution in such an optimization problem is ordinarily chosen from the set of all (weak) Pareto optimal solutions to it. Characterizations of (weak) Pareto optimal solutions for constrained multiobjective programming problems are of practical interest, since multipliers associated with them have useful economic interpretations. Therefore, multiobjective programming has grown remarkably in different directions in the settings of optimality conditions and duality theory in last three decades. Several papers appeared in optimization theory, which concerned Fritz John and Karush-Kuhn-Tucker optimality conditions for multiobjective optimization, but the authors considered the (weak) Pareto case in all of them (see, for example, $2,5,6,14,21,24,26,28,31,34,35$, and others). In [26], by using the Ekeland variational principle, Minami derived generalized conditions of the Fritz-John type given by Clarke's generalized gradient formula, which are necessary for weak Pareto solutions in the considered nondifferentiable vector optimization problem.

Received March 28, 2019; Accepted July 8, 2020.

Communicated by Jein-Shan Chen.

2010 Mathematics Subject Classification. 90C29, 90C46, 90C26, 49J52.

Key words and phrases. nonsmooth mutiobjective programming, vector critical point, (weakly) efficiency, nonsmooth cone- $F J$-pseudo-invexity, nonsmooth cone- $K T$-pseudo-invexity, optimality conditions.

${ }^{*}$ Corresponding author. 
However, in recent years, some of the authors extended the results concerning (weak) efficiency optimality to the more complicated case with arbitrary cones. Craven and Yang [15] introduced generalized cone-invex functions and established a generalized alternative theorem involving such nonsmooth generalized convex functions. Yen and Sach 38 defined the concepts of cone-generalized invexity and cone-nonsmooth invexity for vectorvalued functions with locally Lipschitz components. Giorgi and Guerraggio [19] introduced the concepts of $K$ - $\alpha$-invexity, $K$ - $\alpha$-pseudoinvexity and $K$ - $\alpha$-quasiinvexity in the differentiable case and, using the introduced concepts of generalized convexity, obtained optimality and duality results for considered vector optimization problem over cones in terms of Jacobians of the functions involved. In [36], Suneja et al. defined the concept of $Q$-nonsmooth pseudoinvexity and, by using this concept, they established necessary and sufficient optimality conditions involving Clarke's generalized gradients for the considered multiobjective programming problem with generalized cone constraint. In [9], for solving a class of multiobjective programing problems involving cone-invexity, Chen et al. used the so-called modified objective function method introduced by Antczak [1]. In [3], Antczak proved optimality conditions for proper efficiency for a new class of nonconvex nondifferentiable multiobjective programming problems involving constraints with respect to cones in arbitrary Banach spaces. Recently, under pseudoinvexity hypotheses and via the Clarke generalized Jacobian, Gutiérrez et al. [20] established some relations between several notions of vector critical points and efficient, weakly efficient and ideal efficient solutions of the considered unconstrained vector optimization problem with a locally Lipschitz objective function. Thus, they provided a characterization of (strong) pseudoinvexity through the property that every vector critical point is a weak efficient (and also efficient) solution in the considered unconstrained vector optimization problem. In [37, Tung and Luu established necessary optimality conditions for weakly efficient solutions in terms of the Clarke subdifferentials for the considered nonsmooth multiobjective optimization problem with a cone constraint, an equality constraint and a set constraint.

In this paper, we consider a new class of nonconvex nondifferentiable vector optimization problems with both cone constraint and equality constraint. Each component of the functions constituting the considered vector optimization problem is a locally Lipschitz function defined on a finite-dimensional space and, moreover, the order cone is assumed to be pointed, closed and convex. We define the notions of cone- $F J$-pseudoinvexity and cone- $K T$-pseudoinvexity with respect to cones for a nonsmooth vector optimization problem with both cone inequality constraints and also with equality constraints through the Clarke generalized gradient of a vector-valued function with locally Lipschitz components. We prove that a vector optimization problem with cone inequality and also equality constraints is cone- $F J$-pseudoinvex if and only if every Fritz John vector critical point is a 
global weakly $K$-efficient solution. Further, we also prove that a vector optimization problem with cone inequality and also equality constraints is $K T$-pseudoinvex with respect to cones if and only if every Kuhn-Tucker vector critical point is its global $K$-efficient solution. Thus, we extend the characterization of vectorial critical points to a new class of nonsmooth constrained vector optimization problems with both cone inequality constraint and also equality constraint. Furthermore, we also generalize a lot of previous optimality conditions, concerning the scalar case and the multiobjective Pareto one established for vector optimization problem with cone inequality only. Namely, we generalize the results established by Osuna-Gómez et al. [29] and Arana-Jiménez et al. [4], which were stated in Pareto differentiable problems, and also by Suneja et al. [36], where nondifferentiable vector optimization problems were considered with inequality cone constraints only. Further, we also extend the results established by Gutiérrez et al. 20 for a nonsmooth unconstrained vector optimization problem with a locally Lipschitz objective function to the case a nondifferentiable constrained vector optimization problem with cone inequality and equality constraints. Also we generalize the Fritz John type necessary optimality conditions for a weak Pareto solution in nondifferentiable vector optimization problems with both inequality and equality constraints established by Minami [26]. Namely, we give both Fritz John type and Kuhn-Tucker type necessary optimality conditions for weakly $K$ efficiency in nondifferentiable multiobjective programming problems with cone and equality constraints by the proof which is, in part, as in 26 .

\section{Preliminaries}

In this section, we provide some definitions and some results that we shall use in the sequel.

Let $R_{+}^{k}=\left\{\left(x_{1}, \ldots, x_{k}\right) \in R^{k}: x_{i} \geq 0, i=1, \ldots, k\right\}$. A nonempty subset $K$ of $R^{k}$ is called a cone if $\alpha K \subset K$ for all $\alpha \geq 0$. K is called a convex cone if $K$ is a cone and $K+K \subset K . K$ is called a pointed cone if $K$ is a cone and $K \cap(-K)=\{0\}$ and solid if int $K \neq \emptyset$.

For the closed convex cone $K \subset R^{k}$ with nonempty interior (int $K \neq \emptyset$ ) which induces a partial order in $R^{k}$, we define

(a) $x \leqq_{K} y$ if and only if $y-x \in K$;

(b) $x \leq_{K} y$ if and only if $y-x \in K \backslash\{0\}$;

(c) $x<_{K} y$ if and only if $y-x \in \operatorname{int} K$.

Lemma 2.1. 9, 25] Let $K$ be a convex cone of $R^{k}$ with int $K \neq \emptyset$. Then, for any $x, y \in X$, the following statements are true: 
(i) $y-x \in K$ and $y \in-\operatorname{int} K$ imply $x \in-\operatorname{int} K$;

(ii) $y-x \in K$ and $x \notin-\operatorname{int} K$ imply $y \notin-\operatorname{int} K$;

(iii) $y-x \in K$ and $y \in-K$ imply $x \in-K$;

(iv) $y-x \in-\operatorname{int} K$ and $x \in-\operatorname{int} K$ imply $y \in-\operatorname{int} K$.

Definition 2.2. Let $K \subseteq R^{k}$ be a closed convex cone with nonempty interior and let int $K$ and $\bar{K}$ denote the interior and closure of a cone $K$, respectively. The positive dual cone $K^{+}$of a cone $K$ is defined as

$$
K^{+}:=\left\{y \in R^{k}: y^{T} x \geq 0 \text { for all } x \in K\right\}
$$

Definition 2.3. 7 Let $S$ be a nonempty set of $R^{k}$. The set

$$
S^{0}:=\left\{y \in R^{k}: y^{T} x \geq-1 \text { for all } x \in S\right\}
$$

is called the polar set of $S$.

Now, we re-call the so-called bipolar theorem.

Lemma 2.4. 16 Let $K$ be any closed convex cone and $K^{+}$its dual, and let $x, y \in R^{k}$. Then

$$
x \in K \quad \Longleftrightarrow \quad y^{T} x \geq 0 \quad \text { for all } y \in K^{+}
$$

Furthermore, if int $K \neq \emptyset$, then

$$
x \in \operatorname{int} K \quad \Longleftrightarrow y^{T} x>0 \quad \text { for all } y \in K^{+} \backslash\{0\} .
$$

In [16, it was given the following result.

Corollary 2.5. Let $K$ be any closed convex cone and $K^{+}$its dual, and let $x, y \in R^{k}$. Then (2.2) can be replaced by

$$
x \in K \quad \Longleftrightarrow \quad y^{T} x \geq 0 \quad \text { for all } y \in K^{+},\|y\|=1 \text {. }
$$

Note that 2.2 may be regarded as a simple translation of Farkas' lemma (see [32]) to the language of convex cones.

Lemma 2.6. Let $K$ be a convex cone with int $K \neq \emptyset$ and $K^{+}$be the dual cone of $K$. Then, we have the following:

(1) If $x \in \operatorname{int} K$, then $y^{T} x>0$ for all $y \in K^{+} \backslash\{0\}$. 
(2) If $y \in \operatorname{int} K^{+}$, then $y^{T} x>0$ for all $x \in K \backslash\{0\}$.

Lemma 2.7 (Cartesian product). For closed convex cones $K_{1}$ and $K_{2}$, their Cartesian product $K=K_{1} \times K_{2}$ is a closed convex cone, and $K^{+}=K_{1}^{+} \times K_{2}^{+}$.

A particular case of generating set for a convex cone is given by the following definition.

Definition 2.8. It is said that a nonempty convex subset $B$ of a convex cone $K$ is a base for $K$ if each element $x \in K \backslash\{0\}$ has a unique representation of the form $x=\alpha b$ with $\alpha>0$ and $b \in B$.

As it follows from the above definition, if $K$ has a base, then $K=\operatorname{cone}(B)$, that is, $K=\{\alpha b: \alpha \geq 0, b \in B\}$.

It is well-known, if $B$ is a base for a convex cone, then $0 \notin B$. In particular, a convex cone $K$ which has a base is necessarily pointed.

Now, we give the condition under which dual cones are nontrivial. Such a result was given, for example, by Peressini [30] and Rudin 33.

Lemma 2.9. Let $K \subset R^{k}$ be a closed pointed convex cone and $K^{+}$be the dual cone of $K$. If int $K \neq \emptyset$ and $k_{0} \in \operatorname{int} K, k_{0} \neq 0$, then the set

$$
\mathcal{B}=\left\{b \in K^{+}: b^{T} k_{0}=1\right\}
$$

is a compact convex base for $K^{+}$.

Proof. In order to prove that the set $\mathcal{B}$ is a base of $K^{+}$, we need to prove that the properties of a base are fulfilled. First, note that $0 \notin \mathcal{B}$. Now, we show that any nonzero element of $K^{+}$has a unique representation of the form $\alpha b$ with $\alpha>0$ and $b \in \mathcal{B}$. In order to do this, let $y$ be any nonzero element of $K^{+}$. Then, by (2.3), it follows that $y^{T} x>0$ for every $x \in \operatorname{int} K$. Then, let us take $\alpha=y^{T} k_{0}>0$. Therefore, we define $b:=\alpha^{-1} y$. Hence, $y=\alpha b$. Moreover, we have that $b^{T} k_{0}=1$. Since $b \in K^{+}$and $\alpha=y^{T} k_{0}$, we get that $b \in \mathcal{B}$. Now, suppose that $y=\alpha_{1} b_{1}$ for some $\alpha_{1}>0$ and $b_{1} \in \mathcal{B}$. Then, we have that $\alpha=y^{T} k_{0}=\alpha_{1} b_{1}^{T} k_{0}=\alpha_{1}$, whence $b=b_{1}$. Thus, we have shown that any nonzero element of $K^{+}$has a unique representation of $\alpha b$ with $\alpha>0$ and $b \in \mathcal{B}$. This means by definition that the set $\mathcal{B}$ given by $(2.5)$ is a base of $K^{+}$.

Now, we show that $\mathcal{B}$ is a compact convex base for $K^{+}$. By assumption, $k_{0} \in \operatorname{int} K$, $k_{0} \neq 0$. Hence, the set $\Gamma=K-k_{0}$ is a neighborhood of $0_{n} \in R^{n}$. Then, by definition, (see, for example, [7]), the polar set of $\Gamma$ is defined by

$$
\Gamma^{0}=\left\{y \in R^{k}: y^{T} x \geq-1 \text { for all } x \in \Gamma\right\} .
$$

Hence, by the definition of the set $\Gamma$, we have

$$
\Gamma^{0}=\left\{y \in R^{k}: y^{T} k-y^{T} k_{0} \geq-1 \text { for all } k \in K\right\} .
$$


Thus,

$$
\Gamma^{0}=\left\{y \in R^{+}: y^{T} k \geq y^{T} k_{0}-1 \text { for all } k \in K\right\}
$$

Now, we show that

$$
\Gamma^{0}=\left\{y \in K^{+}: y^{T} k_{0} \leq 1\right\}
$$

We proceed by contradiction. Suppose that there exists $y \notin K^{+}$satisfying the inequality in (2.6). This means that there exists $k \in K$ such that $y^{T} k<0$. Since $K$ is a cone, therefore, the foregoing inequality is also satisfied if it is multiplied by any sufficiently large $\alpha>0$. This means that the value of $y^{T} k$ can be sufficiently small. This is a contradiction to the inequality $y^{T} k \geq y^{T} k_{0}-1$ for all $k \in K$ in which all $y^{T} k$ are bounded below. Then, (2.7) we get if we set $k=0$ in $(2.6)$, which is possible, because any cone contains 0 .

Then, by Banach-Alaoglu Theorem (see, for example, [17, 33]), the polar set of $\Gamma$ is a compact set. Hence, the set $\mathcal{B}$ as a closed subset of $\Gamma^{0}$ is also compact. Moreover, it is obviously a convex set. Thus, $\mathcal{B}$ is a compact convex set not containing the origin. Since we have already proved that the set $\mathcal{B}$ is a base for $K^{+}$, this means that it is a compact convex base for $K^{+}$.

We now re-call a Gordan-type alternative theorem, the so-called theorem of alternatives for linear strict generalized inequalities (see [8,13, 19]).

Theorem 2.10 (Theorem of alternatives for linear strict generalized inequalities). Let $A$ be an element (matrix) of $R^{k \times n}, K^{+}$is the dual cone of $K \subseteq R^{k}$. Then one and only one of the following statements is true:

(i) There exists $x \in R^{n}$ such that $A x<_{K} 0$.

(ii) There exists $y \in K^{+}, y \neq 0$, such that $A^{T} y=0$.

Now, we re-call some definitions and properties for locally Lipschitz functions. Let $X$ be a nonempty subset of $R^{n}$ and $u$ be an arbitrary given point of $X$. Namely, the function $f: X \rightarrow R$ is said to be locally Lipschitz on $X$ if, for every $u \in X$, there exist a neighborhood $U \subset X$ of $u$ and a constant $K_{u}>0$ such that the inequality $\|f(y)-f(z)\| \leq K_{u}\|y-z\|$ holds for all $y, z \in U$.

Definition 2.11. [12] The Clarke generalized directional derivative 12 of a locally Lipschitz function $f: X \rightarrow R$ at $u \in X$ in the direction $v \in R^{n}$, denoted $f^{0}(u ; v)$, is given by

$$
f^{0}(u ; v)=\limsup _{\substack{y \rightarrow u \\ \lambda \downarrow 0}} \frac{f(y+\lambda v)-f(y)}{\lambda} .
$$


Definition 2.12. [12 The Clarke generalized subgradient of a locally Lipschitz function $f: X \rightarrow R$ at $u \in X$, denoted $\partial f(u)$, is the set

$$
\partial f(u)=\left\{y \in R^{n}: f^{0}(u ; v) \geq y^{T} v \text { for all } v \in R^{n}\right\} .
$$

It is well-known that $f^{0}(u ; v)=\max _{y \in \partial f(u)} y^{T} v$ (see 12 ).

Lemma 2.13. [12] Let $f: X \rightarrow R$ be a locally Lipschitz function on a nonempty set $X \subset R^{n}, u$ be an arbitrary point of $X$ and $\alpha \in R$. Then $\partial(\alpha f)(u)=\alpha \partial f(u)$.

Corollary 2.14. Let $f: X \rightarrow R$ be a locally Lipschitz function on a nonempty open set $X \subset R^{n}, u$ be an arbitrary point of $X$. Then $\partial(-f)(u)=-\partial f(u)$.

Proposition 2.15. [12] Let $f_{i}: X \rightarrow R, i=1, \ldots, k$, be locally Lipschitz functions on a nonempty set $X \subset R^{n}, u$ be an arbitrary point of $X \subset R^{n}$. Then

$$
\partial\left(\sum_{i=1}^{k} f_{i}\right)(u) \subseteq \sum_{i=1}^{k} \partial f_{i}(u) .
$$

Equality holds in the above relation if all but at most one of the functions $f_{i}$ is strictly differentiable at $u$.

Corollary 2.16. 12] For any scalars $\alpha_{i}$, one has

$$
\partial\left(\sum_{i=1}^{k} \alpha_{i} f_{i}\right)(u) \subseteq \sum_{i=1}^{k} \alpha_{i} \partial f_{i}(u),
$$

and equality holds if all but at most one of the $f_{i}$ is strictly differentiable at $u$.

Proposition 2.17. [11] Let $U$ be a sequentially compact space, and let $g: R^{n} \times U \rightarrow R$ have the following properties:

(a) $g(x, u)$ is upper semi-continuous in $(x, u)$,

(b) $g$ is locally Lipschitz in $x$, uniformly for $u$ in $U$,

(c) $g_{x}^{0}(x, u, \cdot)=g_{x}^{\prime}(x, u, \cdot)$, where $g_{x}^{\prime}(x, u, \cdot)$ denotes the usual one-sided directional derivative of $g$,

(d) $\partial g_{x}(x, u)$ is upper semi-continuous in $(x, u)$.

Then, the function $f: R^{n} \rightarrow R$ defined by $f(x):=\max _{u \in U} g(x, u)$ is also locally Lipschitz at any $x \in R^{n}$. In addition,

$$
\partial f(x)=\operatorname{conv}\left\{\partial g_{x}(x, u): u \in I(x)\right\},
$$

where $I(x):=\{u \in U: g(x, u)=f(x)\}$. 
Corollary 2.18. Let I be a nonempty index set (not necessarily finite). Further, let the functions $f_{i}: R^{n} \rightarrow R, i \in I$, be locally Lipschitz at a point $\bar{x} \in R^{n}$ and the function $f: R^{n} \rightarrow R$ be defined by $f(x):=\max _{i \in I} f_{i}(x)$. If $I(\bar{x}):=\left\{i \in I: f(\bar{x})=f_{i}(\bar{x})\right\}=\left\{i_{0}\right\}$, then $\partial f(\bar{x})=\partial f_{i_{0}}(\bar{x})$.

Theorem 2.19. [12] Let the function $f: R^{n} \rightarrow R$ be locally Lipschitz at a point $\bar{x} \in R^{n}$ and attain its (local) minimum at $\bar{x}$. Then $0 \in \partial f(\bar{x})$.

Now, we present the definition of the Clarke generalized subgradient of a vector-valued function such that each its component is locally Lipschitz. Let $f: X \rightarrow R^{k}$ be a vectorvalued function such that each its component $f_{i}, i=1, \ldots, k$, is a locally Lipschitz function on $X$.

Definition 2.20. 36 The Clarke generalized subgradient of a function $f=\left(f_{1}, \ldots, f_{k}\right)$ : $X \rightarrow R^{k}$ at $u \in X$ is the set

$$
\partial f(u)=\partial f_{1}(u) \times \cdots \times \partial f_{k}(u)
$$

where $\partial f_{i}(u)$ is the generalized gradient of $f_{i}, i=1, \ldots, k$, at $u$.

In the next theorem, we re-call the Ekeland's variational principle (see [18, 26]).

Theorem 2.21. Let $F: R^{n} \rightarrow R$ be lower semicontinuous and bounded below on $R^{n}$. If $u \in R^{n}$ satisfies the inequality

$$
F(u) \leq \inf _{x \in R^{n}} F(x)+\epsilon
$$

for some $\epsilon>0$, then there exists $z \in R^{n}$ such that

$$
\|u-z\| \leq \sqrt{\epsilon}, \quad F(z) \leq F(u)
$$

and the inequality

$$
F(x)>F(z)-\sqrt{\epsilon}\|x-z\|
$$

holds for all $x \in R^{n}, x \neq z$.

3. Relationships between solutions of the considered vector optimization problem and its vector critical points

In this section, we define a nondifferentiable vector optimization problem with both cone constraints and equality constraints and study some relationships between its (weakly) $K$-efficient solutions and its vector critical points. 
In the paper, we consider the following nondifferentiable multiobjective programming problem:

$$
K \text {-minimize } f(x) \text { subject to }-g(x) \in C, h(x)=0_{p},
$$

where $f: R^{n} \rightarrow R^{k}, g: R^{n} \rightarrow R^{m}, h: R^{n} \rightarrow R^{p}$, each component of the functions mentioned above is locally Lipschitz on $R^{n}, K$ and $C$ are pointed closed convex cones with nonempty interiors in $R^{k}$ and $R^{m}$, respectively.

Let $\Omega:=\left\{x \in R^{n}:-g(x) \in C, h(x)=0_{p}\right\}$ be the set of all feasible solutions in the problem (3.1).

Remark 3.1. If $K=R_{+}^{k}$, then we have a multiobjective Pareto problem. In the case when $K=R_{+}$and $m=1$, the problem (3.1) reduces to a scalar optimization problem.

Definition 3.2. A feasible point $\bar{x}$ is said to be a weakly $K$-efficient solution of the problem (3.1) if and only if there is no other $x \in \Omega$ such that $f(x)-f(\bar{x}) \in-\operatorname{int} K$.

Definition 3.3. A feasible point $\bar{x}$ is said to be a $K$-efficient solution of the problem (3.1) if and only if there is no other $x \in \Omega$ such that $f(x)-f(\bar{x}) \in-K \backslash\{0\}$.

Now, we introduce the definition of a vector critical point in the sense of Fritz John (or shortly, a vector Fritz John critical point) for the considered multiobjective programming problem (3.1).

Definition 3.4. Let $\bar{x}$ be a feasible solution of the problem (3.1). If there exist $\bar{\lambda} \in K^{+}$, $\bar{\mu} \in C^{+}$and $\bar{\vartheta} \in R^{q}$, not all zero, such that

$$
0_{n} \in \bar{\lambda}^{T} \partial f(\bar{x})+\bar{\mu}^{T} \partial g(\bar{x})+\bar{\vartheta}^{T} \partial h(\bar{x})
$$

then $\bar{x}$ is said to be a vector Fritz John critical point or, in other words, a vector critical point in the sense of Fritz John (with Lagrange multipliers $\bar{\lambda} \in K^{+}, \bar{\mu} \in C^{+}$and $\bar{\vartheta} \in R^{p}$ ) for the considered multiobjective programming problem (3.1).

In other words, relation (3.2) means that, there exist $A \in \partial f(\bar{x}), B \in \partial g(\bar{x}), D \in \partial h(\bar{x})$ such that $\bar{\lambda}^{T} A+\bar{\mu}^{T} B+\bar{\vartheta}^{T} D=0_{n}$.

Now, we prove the Fritz John necessary optimality condition for a feasible solution $\bar{x}$ to be a weakly $K$-efficient solution of the considered multiobjective programming problem (3.1). In this way, we generalize a similar result established by Minami [26] for weak Pareto-optimal solutions of nondifferentiable multicriteria optimization problems to the case of weakly $K$-efficient solutions in vector optimization problems with cone constraints.

Theorem 3.5. Let $\bar{x} \in \Omega$ be a weakly $K$-efficient solution of the considered nondifferentiable multiobjective programming problem (3.1). Further, we assume that the functions 
constituting (3.1) are regular in the sense of Clarke (see [12, Definition 2.3.4]). Then $\bar{x}$ is also a vector Fritz John critical point of the considered multiobjective programming problem (3.1), that is, there exist $\bar{\lambda} \in K^{+}, \bar{\mu} \in C^{+}$and $\bar{\vartheta} \in R^{p}$, not all zero, such that the relation $(3.2)$ is satisfied.

Proof. By assumption, $\bar{x} \in \Omega$ is a weakly $K$-efficient solution of the problem (3.1). Then, by Definition 3.2 , the relation

$$
f(x)-f(\bar{x}) \notin-\operatorname{int} K
$$

holds for all $x \in \Omega$. Let us denote $\xi=(\lambda, \mu, \vartheta) \in K^{+} \times C^{+} \times R^{p}$ such that $\|\xi\|=1$. Further, let us pick $k_{0} \in \operatorname{int} K$ such that

$$
0<\max _{\substack{(\lambda, \mu, \vartheta) \in K^{+} \times C^{+} \times R^{p} \\\|(\lambda, \mu, \vartheta)\|=1}} \lambda^{T} k_{0} \leq 1
$$

The existence of such an element $k_{0} \in \operatorname{int} K$ follows from the fact that $K$ is a pointed closed convex cone with a nonempty interior. Then, for any $\epsilon>0$, we define the following function

$$
F_{\epsilon}(x)=\max _{\substack{(\lambda, \mu, \vartheta) \in K^{+} \times C^{+} \times R^{p} \\\|(\lambda, \mu, \vartheta)\|=1}}\left\{\lambda^{T}\left(f(x)-f(\bar{x})+\epsilon k_{0}\right)+\mu^{T} g(x)+\vartheta^{T} h(x)\right\} .
$$

Note that $F_{\epsilon}$ is a locally Lipschitz function.

Now, we prove that $F_{\epsilon}(x)>0$ for any $\epsilon>0$ and all $x \in R^{n}$. We proceed by contradiction. Suppose, contrary to the result, that $F_{\epsilon_{0}}\left(x_{0}\right) \leq 0$ for some $\epsilon_{0}>0$ and some $x_{0} \in R^{n}$. We now show that, for all $(\lambda, \mu, \vartheta) \in K^{+} \times C^{+} \times R^{p}$ and $\|(\lambda, \mu, \vartheta)\|=1, \mu^{T} g\left(x_{0}\right) \leq 0$, $\vartheta^{T} h\left(x_{0}\right)=0$ and, moreover, $\lambda^{T}\left(f\left(x_{0}\right)-f(\bar{x})\right)<0$.

In fact, let us take $\xi \in K^{+} \times C^{+} \times R^{p},\|\xi\|=1$ such that $\xi=(0,0, \vartheta)$. Then, $\vartheta \in R^{p}$, $\vartheta \neq 0$ and, moreover,

$$
\begin{aligned}
& \max _{\substack{(0,0, \vartheta) \in K^{+} \times C^{+} \times R^{p} \\
\|(\lambda, \mu, \vartheta)\|=1}}\left\{\vartheta^{T} h\left(x_{0}\right)\right\} \\
& \leq \max _{\substack{(\lambda, \mu, \vartheta) \in K^{+} \times C^{+} \times R^{p} \\
\|(\lambda, \mu, \vartheta)\|=1}}\left\{\lambda^{T}\left(f\left(x_{0}\right)-f(\bar{x})+\epsilon k_{0}\right)+\mu^{T} g\left(x_{0}\right)+\vartheta^{T} h\left(x_{0}\right)\right\} \leq 0 .
\end{aligned}
$$

This implies that $\vartheta^{T} h\left(x_{0}\right)=0$. Thus, by $\vartheta \neq R^{p} \backslash\{0\}$ and $\vartheta^{T} h\left(x_{0}\right)=0$, we conclude that $h\left(x_{0}\right)=0$.

Now, let us take $\xi \in K^{+} \times C^{+} \times R^{p},\|\xi\|=1$ such that $\xi=(0, \mu, 0)$. Then, $\mu \in C^{+} \backslash\{0\}$ 
and, moreover,

$$
\begin{aligned}
& \max _{\substack{(0, \mu, 0) \in K^{+} \times C^{+} \times R^{p} \\
\|(\lambda, \mu, \vartheta)\|=1}}\left\{\mu^{T} g\left(x_{0}\right)\right\} \\
& \leq \max _{\substack{(\lambda, \mu, \vartheta) \in K^{+} \times C^{+} \times R^{p} \\
\|(\lambda, \mu, \vartheta)\|=1}}\left\{\lambda^{T}\left(f\left(x_{0}\right)-f(\bar{x})+\epsilon k_{0}\right)+\mu^{T} g\left(x_{0}\right)+\vartheta^{T} h\left(x_{0}\right)\right\} \leq 0 .
\end{aligned}
$$

Thus, we conclude by $\mu \in C^{+} \backslash\{0\}$ and $\mu^{T} g\left(x_{0}\right) \leq 0$ using 2.4 that $g\left(x_{0}\right) \in-C$. Hence, by $g\left(x_{0}\right) \in-C$ and $h\left(x_{0}\right)=0$, we conclude that $x_{0} \in \Omega$.

Now, let us take $\xi \in K^{+} \times C^{+} \times R^{p},\|\xi\|=1$ such that $\xi=(\lambda, 0,0)$. Then, $\lambda \in K^{+} \backslash\{0\}$ and, moreover,

$$
\begin{aligned}
& \max _{\substack{(\lambda, 0,0) \in K^{+} \times C^{+} \times R^{p} \\
\|(\lambda, \mu, \vartheta)\|=1}}\left\{\lambda^{T}\left(f\left(x_{0}\right)-f(\bar{x})+\epsilon k_{0}\right)\right\} \\
\leq & \max _{\substack{(\lambda, \mu, \vartheta) \in K^{+} \times C^{+} \times R^{p} \\
\|(\lambda, \mu, \vartheta)\|=1}}\left\{\lambda^{T}\left(f\left(x_{0}\right)-f(\bar{x})+\epsilon k_{0}\right)+\mu^{T} g\left(x_{0}\right)+\vartheta^{T} h\left(x_{0}\right)\right\} \leq 0 .
\end{aligned}
$$

Hence, we get that $\lambda^{T}\left(f\left(x_{0}\right)-f(\bar{x})+\epsilon k_{0}\right) \leq 0$. Since $\epsilon>0, k_{0} \in \operatorname{int} K$ and $\lambda \in K^{+}, \lambda \neq 0$, by Lemma 2.6, we have that $\lambda^{T}\left(f\left(x_{0}\right)-f(\bar{x})\right)<0$. Further, since $\lambda^{T}\left(f\left(x_{0}\right)-f(\bar{x})\right)<0$ and $\lambda \in K^{+} \backslash\{0\}$, by (2.3), it follows that $f\left(x_{0}\right)-f(\bar{x}) \in-\operatorname{int} K$. Since $x_{0} \in \Omega$, we have a contradiction to the assumption that $\bar{x}$ is a weakly $K$-efficient solution of the considered nondifferentiable multiobjective programming problem (3.1). Hence, we have shown that $F_{\epsilon}(x)>0$ for all $\epsilon>0$ and all $x \in R^{n}$.

This means that the function $F_{\epsilon}$ is bounded below for each $\epsilon>0$. Thus, by (3.3) and (3.4), we have that, for any $x \in R^{n}$,

$$
F_{\epsilon}(\bar{x}) \leq \inf _{x \in R^{n}} F_{\epsilon}(x)+\epsilon
$$

Then, since $F_{\epsilon}$ satisfies all assumptions of Theorem 2.21, there exists $z_{\epsilon} \in R^{n}$ such that

$$
\left\|\bar{x}-z_{\epsilon}\right\| \leq \sqrt{\epsilon}
$$

and the inequality

$$
F_{\epsilon}(x)+\sqrt{\epsilon}\left\|x-z_{\epsilon}\right\|>F_{\epsilon}\left(z_{\epsilon}\right)
$$

holds for all $x \in R^{n}, x \neq z_{\epsilon}$. Note that the function $F_{\epsilon}(\cdot)+\sqrt{\epsilon}\left\|\cdot-z_{\epsilon}\right\|$ is locally Lipschitz. Moreover, by (3.6), it follows that $z_{\epsilon}$ is a global minimizer of the function $F_{\epsilon}(\cdot)+\sqrt{\epsilon}\left\|\cdot-z_{\epsilon}\right\|$ over $R^{n}$. Hence, by Theorem 2.19 , we have that

$$
0 \in \partial\left(F_{\epsilon}(x)+\sqrt{\epsilon}\left\|x-z_{\epsilon}\right\|\right)\left(z_{\epsilon}\right) .
$$


Thus, by Corollary 2.16, it follows that

$$
\partial\left(F_{\epsilon}(x)+\sqrt{\epsilon}\left\|x-z_{\epsilon}\right\|\right)\left(z_{\epsilon}\right) \subseteq \partial F_{\epsilon}\left(z_{\epsilon}\right)+\sqrt{\epsilon} B(0,1)
$$

where $B(0,1)$ is the unit ball in $R^{n}$. Combining (3.7) and $(3.8)$, we get

$$
0 \in \partial F_{\epsilon}\left(z_{\epsilon}\right)+\sqrt{\epsilon} B(0,1)
$$

Now, we have to calculate the subdifferential of $F_{\epsilon}$ at $z_{\epsilon}$. In order to do it, let us denote $\Gamma_{\epsilon}(x)=\left(f(x)-f(\bar{x})+\epsilon k_{0}, g(x), h(x)\right)$ for $\epsilon \geq 0$. Hence, by (3.4), we have

$$
F_{\epsilon}(x)=\max _{\substack{\xi \in K^{+} \times C^{+} \times R^{p} \\\|\xi\|=1}} \xi^{T} \Gamma_{\epsilon}(x)
$$

We have already shown that $F_{\epsilon}(x)>0$ for all $x \in R^{n}$. Therefore, $F_{\epsilon}\left(z_{\epsilon}\right)>0$. Then, note that the linear function $\xi \rightarrow \xi^{T} \Gamma_{\epsilon}\left(z_{\epsilon}\right)$ attains a maximum at some point $\xi_{\epsilon}=\left(\lambda_{\epsilon}, \mu_{\epsilon}, \vartheta_{\epsilon}\right)$ on the set $\left\{\xi=(\lambda, \mu, \vartheta) \in K^{+} \times C^{+} \times R^{p}:\|\xi\|=1\right\}$. Thus, we have

$$
\max _{\substack{\xi \in K^{+} \times C^{+} \times R^{p} \\\|\xi\|=1}} \xi^{T} \Gamma_{\epsilon}\left(z_{\epsilon}\right)=\xi_{\epsilon}^{T} \Gamma_{\epsilon}\left(z_{\epsilon}\right) .
$$

Moreover, $\xi_{\epsilon}$ is a unique point at which this function attains a maximum. Therefore, by (3.10), (3.11) and Corollary 2.18, we get

$$
\partial F_{\epsilon}\left(z_{\epsilon}\right)=\partial\left(\max _{\substack{\xi \in K^{+} \times C^{+} \times R^{p} \\\|\xi\|=1}} \xi^{T} \Gamma_{\epsilon}\left(z_{\epsilon}\right)\right)=\partial\left(\left(\xi_{\epsilon}\right)^{T} \Gamma_{\epsilon}\right)\left(z_{\epsilon}\right) .
$$

Hence, using the above introduced denotations, we obtain

$$
\partial\left(\left(\xi_{\epsilon}\right)^{T} \Gamma_{\epsilon}\right)\left(z_{\epsilon}\right)=\partial\left(\left(\lambda_{\epsilon}\right)^{T} f+\left(\mu_{\epsilon}\right)^{T} g+\left(\vartheta_{\epsilon}\right)^{T} h\right)\left(z_{\epsilon}\right) .
$$

Thus, by (3.5), it follows that $z_{\epsilon} \rightarrow \bar{x}$ as $\epsilon \rightarrow 0$. Also we have in such a case that $\Gamma\left(z_{\epsilon}\right) \rightarrow$ $(0, g(\bar{x}), h(\bar{x}))$ and, moreover, $\xi_{\epsilon}$ tends to some $\bar{\xi}=(\bar{\lambda}, \bar{\mu}, \bar{\vartheta}) \in\{(\lambda, \mu, \vartheta):\|(\lambda, \mu, \vartheta)\|=1\}$ (taking some subsequence, if it is necessary). By the definition of $\Gamma_{\epsilon}$, it follows that $\Gamma_{0}(x)=(f(x)-f(\bar{x}), g(x), h(x))$. Then, note that $\partial\left(\left(\xi_{\epsilon}\right)^{T} \Gamma_{\epsilon}\right)\left(z_{\epsilon}\right)=\partial\left(\left(\xi_{\epsilon}\right)^{T} \Gamma_{0}\right)\left(z_{\epsilon}\right)$. As it follows from [12, Proposition 2.1.5], $x \rightarrow \partial \Gamma_{0}(x)$ is an upper semicontinuous function at $\bar{x}$. Hence, $(\xi, x) \rightarrow \partial\left(\xi^{T} \Gamma_{0}\right)(x)$ is also an upper semicontinuous function at $(\bar{\xi}, \bar{x})$. Then, by $(3.9),(3.12)$ and $(3.13)$, it follows that

$$
0 \in \partial\left(\bar{\xi}^{T} \Gamma_{0}\right)(\bar{x})=\partial\left(\bar{\lambda}^{T} f+\bar{\mu}^{T} g+\bar{\vartheta}^{T} h\right)(\bar{x}) .
$$

Hence, by Corollary 2.16, (3.14) gives $(3.2)$. This completes the proof of this theorem. 
Now, we introduce the definition of a vector critical point in the sense of Kuhn-Tucker (or shortly, a vector Kuhn-Tucker critical point) for the considered multiobjective programming problem 3.1 .

Definition 3.6. Let $\bar{x}$ be a feasible solution in problem (3.1). If there exist $\bar{\lambda} \in K^{+} \backslash\{0\}$, $\bar{\mu} \in C^{+}$and $\bar{\vartheta} \in R^{p}$ such that

$$
0_{n} \in \bar{\lambda}^{T} \partial f(\bar{x})+\bar{\mu}^{T} \partial g(\bar{x})+\bar{\vartheta}^{T} \partial h(\bar{x})
$$

then $\bar{x}$ is said to be a vector Kuhn-Tucker critical point or, in other words, a vector critical point in the sense of Kuhn-Tucker (with Lagrange multipliers $\bar{\lambda} \in K^{+} \backslash\{0\}, \bar{\mu} \in C^{+}$and $\bar{\vartheta} \in R^{p}$ ) for the considered multiobjective programming problem (3.1).

Remark 3.7. In other words, condition (3.15) means that there exist $A \in \partial f(\bar{x}), B \in \partial g(\bar{x})$, $D \in \partial h(\bar{x})$ such that $\bar{\lambda}^{T} A+\bar{\mu}^{T} B+\bar{\vartheta}^{T} D=0_{n}$.

Now, we present the Kuhn-Tucker necessary optimality condition for a feasible solution $\bar{x}$ to be a weakly $K$-efficient solution of the considered multiobjective programming problem (3.1). Before proving this result, we give the constraint qualification (NMFCQ) which is a nonsmooth version of the Mangasarian-Fromovitz constraint qualification.

Constraint Qualification (NMFCQ). It is said that the nonsmooth MangasarianFromovitz constraint qualification (NMFCQ) holds at $\bar{x} \in \Omega$ for $(3.1)$ if there exists $d \in R^{n}$ such that

(a) $B_{j}^{T} d<0, \forall B_{j} \in \partial g_{j}(\bar{x}), j=1, \ldots, m$,

(b) $D^{T} d=0, \forall D \in \partial h(\bar{x})$,

(c) for each $D \in \partial h(\bar{x})$, the rows of $D$ are linearly independent.

Theorem 3.8. Let $\bar{x} \in \Omega$ be a weakly $K$-efficient solution of the considered nondifferentiable multiobjective programming problem (3.1) and the functions constituting (3.1) be regular in the sense of Clarke. Further, we assume that the constraint qualification (NMFCQ) holds at $\bar{x}$ for (3.1). Then $\bar{x}$ is also a vector Kuhn-Tucker critical point of the considered multiobjective programming problem (3.1), that is, there exist $\bar{\lambda} \in K^{+} \backslash\{0\}$, $\bar{\mu} \in C^{+}$and $\bar{\vartheta} \in R^{p}$ such that the relation (3.15) is satisfied.

Remark 3.9. In [36], Suneja et al. considered a nonsmooth vector optimization problem with cone constraints only. For such nondifferentiable vector optimization problems, under the concept of nonsmooth $K$-generalized invexity, they established Fritz John type and Kuhn-Tucker type necessary optimality conditions for a nonsmooth vector minimization problem by using the Generalized Alternative Theorem given by Craven and Yang [15]. Further, by using the nonsmooth $K$-generalized invexity notion and also utilizing new 
concepts of nonsmooth $K$-quasi-invexity and (strictly or strongly) nonsmooth $K$-pseudoinvexity, Suneja et al. [36] also proved a number of sufficient optimality conditions for a nonsmooth vector optimization problem wherein Clarke's generalized gradient is used.

Further, note that we consider a more general nondifferentiable multiobjective programming problem than the one in [36]. Namely, we analyze optimality results also for a nonsmooth vector optimization problem with both cone and equality constraints in the opposite to a nonsmooth vector optimization problem with only cone constraints considered by Suneja et al. [36]. In fact, we have derived new characterizations of a vector critical point in the Fritz John sense and a vector critical point in the sense of Kuhn-Tucker for such a nonsmooth vector optimization problems. We have proved in Theorem 3.5 the conditions for a weakly $K$-efficient solution to be a vector Fritz John critical point in the considered nonsmooth vector optimization problem with both cone and equality constraints. Note that we haven't used any generalized convexity concept in proving these results, in opposition to the similar results earlier established, for example, in [10,36].

Note that Fritz John and Kuhn-Tucker critical points provide necessary conditions to locate weakly $K$-efficient solutions of the considered multiobjective programming problem (3.1), but these conditions are not sufficient. Therefore, in order to ensure the sufficiency of the necessary optimality conditions mentioned above, we introduce new concepts of generalized convexity for the considered nondifferentiable vector optimization problem with both cone and equality constraints, namely, the notions of cone- $F J$-pseudoinvexity and cone- $K T$-pseudoinvexity.

Definition 3.10. Let $T_{-}$and $T_{+}$be sets of indices (one of them can be empty) such that $T_{-} \cap T_{+}=\emptyset, T_{-} \cup T_{+}=\{1, \ldots, p\}$ and $\bar{x} \in \Omega$. If there exists $\eta: \Omega \times \Omega \rightarrow R^{n}$ such that the following relation

$$
\left\{\begin{array} { l } 
{ f ( x ) - f ( \overline { x } ) \in - \operatorname { i n t } K , } \\
{ - g ( \overline { x } ) \in C , } \\
{ h ( \overline { x } ) = 0 _ { p } }
\end{array} \Longrightarrow \left\{\begin{array}{ll}
A \eta(x, \bar{x}) \in-\operatorname{int} K, \\
B \eta(x, \bar{x}) \in-\operatorname{int} C, \\
-D_{t} \eta(x, \bar{x}) \in-\operatorname{int} R_{+}, \quad \forall t \in T_{-}, \\
D_{t} \eta(x, \bar{x}) \in-\operatorname{int} R_{+}, \quad \forall t \in T_{+}
\end{array}\right.\right.
$$

holds for all $x \in \Omega$ and every $A \in \partial f(\bar{x}), B \in \partial g(\bar{x}), D_{t} \in \partial h_{t}(\bar{x}), t \in T_{-} \cup T_{+}$, then the problem (3.1) is said to be $\left(K \times C \times R_{+}^{r} \times R_{+}^{w}\right)$-FJ-pseudoinvex at the point $\bar{x}$ on $\Omega$, where $r=\left|T_{-}\right|$and $w=\left|T_{+}\right|$.

If the relation $(3.16)$ is satisfied at each $\bar{x} \in \Omega$, then the problem $(3.1)$ is said to be $\left(K \times C \times R_{+}^{r} \times R_{+}^{w}\right)-F J$-pseudoinvex on $\Omega$, where $r=\left|T_{-}\right|$and $w=\left|T_{+}\right|$.

Remark 3.11. If the relation (3.16) is satisfied at $\bar{x} \in \Omega$ on $\Omega$ for some $r$ and $w$ with $r+w=p$, then the problem (3.1) is said to be $\left(K \times C \times R_{+}^{p}\right)-F J$-pseudoinvex at the point $\bar{x}$ on $\Omega$. 
Remark 3.12. If the set $T_{-}$is empty, then 3.16 reduces to the relation

$$
\left\{\begin{array} { l } 
{ f ( x ) - f ( \overline { x } ) \in - \operatorname { i n t } K , } \\
{ - g ( \overline { x } ) \in C , } \\
{ h ( \overline { x } ) = 0 _ { p } }
\end{array} \Longrightarrow \left\{\begin{array}{l}
A \eta(x, \bar{x}) \in-\operatorname{int} K \\
B \eta(x, \bar{x}) \in-\operatorname{int} C \\
D \eta(x, \bar{x}) \in-\operatorname{int} R_{+}^{p}
\end{array}\right.\right.
$$

which holds for all $x \in \Omega$ and every $A \in \partial f(\bar{x}), B \in \partial g(\bar{x}), D \in \partial h(\bar{x})$. In such a case, the problem $(3.1)$ is said to be $\left(K \times C \times R_{+}^{p}\right)-F J^{+}$-pseudoinvex at the point $\bar{x}$ on $\Omega$.

Remark 3.13. If the set $T_{+}$is empty, then 3.16 reduces to the relation

$$
\left\{\begin{array} { l } 
{ f ( x ) - f ( \overline { x } ) \in - \operatorname { i n t } K , } \\
{ - g ( \overline { x } ) \in C , } \\
{ h ( \overline { x } ) = 0 _ { p } }
\end{array} \Longrightarrow \left\{\begin{array}{l}
A \eta(x, \bar{x}) \in-\operatorname{int} K \\
B \eta(x, \bar{x}) \in-\operatorname{int} C \\
-D \eta(x, \bar{x}) \in-\operatorname{int} R_{+}^{p}
\end{array}\right.\right.
$$

which holds for all $x \in \Omega$ and every $A \in \partial f(\bar{x}), B \in \partial g(\bar{x}), D \in \partial h(\bar{x})$. In such a case, the problem $(3.1)$ is said to be $\left(K \times C \times R_{+}^{p}\right)-F J^{-}$-pseudoinvex at the point $\bar{x}$ on $\Omega$.

Definition 3.14. Let $T_{-}$and $T_{+}$be sets of indices (one of them can be empty) such that $T_{-} \cap T_{+}=\emptyset, T_{-} \cup T_{+}=\{1, \ldots, p\}$ and $\bar{x} \in \Omega$. If there exists $\eta: \Omega \times \Omega \rightarrow R^{n}$ such that the relation

$$
\left\{\begin{array} { l } 
{ f ( x ) - f ( \overline { x } ) \in - K \backslash \{ 0 \} , } \\
{ - g ( \overline { x } ) \in C , } \\
{ h ( \overline { x } ) = 0 _ { p } }
\end{array} \Longrightarrow \left\{\begin{array}{ll}
A \eta(x, \bar{x}) \in-\operatorname{int} K, \\
B \eta(x, \bar{x}) \in-\operatorname{int} C, \\
-D_{t} \eta(x, \bar{x}) \in-\operatorname{int} R_{+}, \quad \forall t \in T_{-}, \\
D_{t} \eta(x, \bar{x}) \in-\operatorname{int} R_{+}, \quad \forall t \in T_{+}
\end{array}\right.\right.
$$

holds for all $x \in \Omega$ and every $A \in \partial f(\bar{x}), B \in \partial g(\bar{x}), D_{t} \in \partial h_{t}(\bar{x}), t \in T_{-} \cup T_{+}$, then the problem (3.1) is said to be $\left(K \times C \times R_{+}^{r} \times R_{+}^{w}\right)$ - $K T$-pseudoinvex at the point $\bar{x} \in \Omega$ on $\Omega$, where $r=\left|T_{-}\right|$and $w=\left|T_{+}\right|$.

If the relation (3.17) is satisfied at each $\bar{x} \in \Omega$, then the problem (3.1) is said to be $\left(K \times C \times R_{+}^{r} \times R_{+}^{w}\right)$-KT-pseudoinvex on $\Omega$, where $r=\left|T_{-}\right|$and $w=\left|T_{+}\right|$.

Remark 3.15. If the relation (3.16) is satisfied at $\bar{x} \in \Omega$ on $\Omega$ for some $r$ and $w$ with $r+w=p$, then the problem $(3.1)$ is said to be $\left(K \times C \times R_{+}^{p}\right)-K T$-pseudoinvex at the point $\bar{x}$ on $\Omega$.

Remark 3.16. If the set $T_{-}$is empty, then 3.17 reduces to the relation

$$
\left\{\begin{array} { l } 
{ f ( x ) - f ( \overline { x } ) \in - K \backslash \{ 0 \} , } \\
{ - g ( \overline { x } ) \in C , } \\
{ h ( \overline { x } ) = 0 _ { p } }
\end{array} \quad \Longrightarrow \quad \left\{\begin{array}{l}
A \eta(x, \bar{x}) \in-\operatorname{int} K, \\
B \eta(x, \bar{x}) \in-\operatorname{int} C \\
D \eta(x, \bar{x}) \in-\operatorname{int} R_{+}^{p}
\end{array}\right.\right.
$$


which holds for all $x \in \Omega$ and every $A \in \partial f(\bar{x}), B \in \partial g(\bar{x}), D \in \partial h(\bar{x})$. In such a case, the problem (3.1) is said to be $\left(K \times C \times R_{+}^{p}\right)$ - $K T^{+}$-pseudoinvex at the point $\bar{x}$ on $\Omega$.

Remark 3.17. If the set $T_{+}$is empty, then 3.17 reduces to the relation

$$
\left\{\begin{array} { l } 
{ f ( x ) - f ( \overline { x } ) \in - K \backslash \{ 0 \} , } \\
{ - g ( \overline { x } ) \in C , } \\
{ h ( \overline { x } ) = 0 _ { p } }
\end{array} \quad \Longrightarrow \quad \left\{\begin{array}{l}
A \eta(x, \bar{x}) \in-\operatorname{int} K, \\
B \eta(x, \bar{x}) \in-\operatorname{int} C, \\
-D \eta(x, \bar{x}) \in-\operatorname{int} R_{+}^{p}
\end{array}\right.\right.
$$

which holds for all $x \in \Omega$ and every $A \in \partial f(\bar{x}), B \in \partial g(\bar{x}), D \in \partial h(\bar{x})$. In such a case, the problem $(3.1)$ is said to be $\left(K \times C \times R_{+}^{p}\right)$ - $K T^{-}$-pseudoinvex at the point $\bar{x}$ on $\Omega$.

In order to prove both the necessity and sufficiency for (weakly) $K$-efficiency, we utilize the concept of nonsmooth generalized convexity defined above. Further, for each $\vartheta \in R^{p}$, let us introduce the following denotations: $T_{+}^{\vartheta}=\left\{t \in\{1, \ldots, p\}: \vartheta_{t} \geq 0\right\}$ and $T_{-}^{\vartheta}=\{t \in$ $\left.\{1, \ldots, p\}: \vartheta_{t}<0\right\}$. Note that one of the sets $T_{+}^{\vartheta}$ and $T_{-}^{\vartheta}$ may be empty.

Theorem 3.18. Let $\bar{x} \in \Omega$ be a vector Fritz John critical point with Lagrange multipliers $\bar{\lambda} \in K^{+}, \bar{\mu} \in C^{+}$and $\bar{\vartheta} \in R^{p}$ with $\bar{\lambda}$ and $\bar{\mu}$ not both equal to 0 . Further, let $T_{+}^{\bar{\vartheta}}=$ $\left\{t \in\{1, \ldots, p\}: \bar{\vartheta}_{t} \geq 0\right\}$ and $T_{-}^{\bar{\vartheta}}=\left\{t \in\{1, \ldots, p\}: \bar{\vartheta}_{t}<0\right\}$. If we assume that the problem (3.1) is $\left(K \times C \times R_{+}^{r} \times R_{+}^{w}\right)$-FJ-pseudoinvex at $\bar{x}$ on $\Omega$, where $r=\left|T_{-}^{\bar{\vartheta}}\right|, w=\left|T_{+}^{\bar{\vartheta}}\right|$ and $r+w=p$, then $\bar{x}$ is a weakly $K$-efficient solution of the problem (3.1).

Proof. Assume that $\bar{x} \in \Omega$ is a vector Fritz John critical point in problem (3.1) with the Lagrange multipliers $\bar{\lambda} \in K^{+}, \bar{\mu} \in C^{+}$and $\bar{\vartheta} \in R^{p}$. Further, by assumption, the problem (3.1) is $\left(K \times C \times R_{+}^{w} \times R_{+}^{r}\right)$ - $F J$-pseudoinvex at $\bar{x}$ on $\Omega$, where $r=\left|T_{-}^{\bar{\vartheta}}\right|, w=\left|T_{+}^{\bar{\vartheta}}\right|$ and $r+w=p$. This means that relations (3.16) are fulfilled at $\bar{x} \in \Omega$ on $\Omega$ with respect to the same function $\eta$. We show that $\bar{x}$ is a weakly $K$-efficient solution of the problem (3.1). Suppose, contrary to the result, that $\bar{x}$ is not a weakly $K$-efficient solution of the problem (3.1). Then, by Definition 3.2 , there exists $\widetilde{x} \in \Omega$ such that

$$
f(\widetilde{x})-f(\bar{x}) \in-\operatorname{int} K \text {. }
$$

By $\widetilde{x} \in \Omega$ and $\bar{x} \in \Omega$, it follows that

$$
-g(\widetilde{x}) \in C, \quad-g(\bar{x}) \in C, \quad h(\bar{x})=0 .
$$

Hence, by Definition 3.10, (3.18) and 3.19$)$ imply that the relations

$$
\begin{cases}A \eta(\widetilde{x}, \bar{x}) \in-\operatorname{int} K, & \\ B \eta(\widetilde{x}, \bar{x}) \in-\operatorname{int} C, & \\ -D_{t} \eta(\widetilde{x}, \bar{x}) \in-\operatorname{int} R_{+}, \quad \forall t \in T_{r}^{\bar{\vartheta}}, \\ D_{t} \eta(\widetilde{x}, \bar{x}) \in-\operatorname{int} R_{+}, \quad \forall t \in T_{w}^{\vartheta}\end{cases}
$$


hold for any $A \in \partial f(\bar{x}), B \in \partial g(\bar{x}), D_{t} \in \partial h_{t}(\bar{x}), \forall t \in T_{-}^{\bar{\vartheta}}, D_{t} \in \partial h_{t}(\bar{x}), \forall t \in T_{+}^{\bar{\vartheta}}$. Since $\bar{\lambda} \in K^{+}, \bar{\mu} \in C^{+}, \bar{\vartheta}_{-} \in R^{\left|T_{-}^{\bar{\vartheta}}\right|}, \bar{\vartheta}_{+} \in R^{\left|T_{+}^{\bar{\vartheta}}\right|}$ with $\bar{\lambda}$ and $\bar{\mu}$ not both equal to 0 , relations in 3.20 yield that the inequality

$$
\left[\bar{\lambda}^{T} A+\bar{\mu}^{T} B+\bar{\vartheta}^{T} D\right] \eta(\widetilde{x}, \bar{x})<0
$$

holds for any $A \in \partial f(\bar{x}), B \in \partial g(\bar{x}), D_{t} \in \partial h_{t}(\bar{x}), \forall t \in T_{-}^{\bar{\vartheta}}, D_{t} \in \partial h_{t}(\bar{x}), \forall t \in T_{+}^{\bar{\vartheta}}$, which contradicts the assumption that $\bar{x}$ is a vector Fritz John critical point $\bar{x}$ (with Lagrange multipliers $\bar{\lambda} \in K^{+}, \bar{\mu} \in C^{+}$and $\bar{\vartheta} \in R^{p}$ ). This means that $\bar{x}$ is a weakly $K$-efficient solution of the problem (3.1) and completes the proof of this theorem.

Remark 3.19. As it follows from the proof of Theorem 3.18 in order to prove that $\bar{x} \in \Omega$, being a vector Fritz John critical point (with the Lagrange multipliers $\bar{\lambda} \in K^{+}, \bar{\mu} \in C^{+}$ and $\bar{\vartheta} \in R^{p}$ ), is a weakly $K$-efficient solution in the problem (3.1), it is sufficient to assume that the problem 3.1$)$ is $\left(K \times C \times R_{+}^{r} \times R_{+}^{w}\right)-F J$-pseudoinvex at $\bar{x}$ on $\Omega$, where $r=\left|T_{-}^{\bar{\vartheta}}\right|$ and $w=\left|T_{+}^{\bar{\vartheta}}\right|$.

Theorem 3.20. Let every vector Fritz John critical point of the considered multiobjective programming problem (3.1) be its weakly $K$-efficient solution. Then, the problem (3.1) is $\left(K \times C \times R_{+}^{r_{\bar{x}}} \times R_{+}^{w_{\bar{x}}}\right)-F J$-pseudoinvex at each point $\bar{x} \in \Omega$ on $\Omega$, where $r_{\bar{x}}$ and $w_{\bar{x}}$ are any integers such that $r_{\bar{x}}+w_{\bar{x}}=p$. In other words, the problem 3.1 is $\left(K \times C \times R_{+}^{p}\right)-F J$ pseudoinvex on $\Omega$.

Proof. Assume that every vector Fritz John critical point of the problem (3.1) is its $K$ weakly efficient solution. Let $\bar{x}$ be any feasible solution in the problem $(3.1)$. We show that the problem $(3.1)$ is $\left(K \times C \times R_{+}^{r_{\bar{x}}} \times R_{+}^{w_{\bar{x}}}\right)-F J$-pseudoinvex at each point $\bar{x} \in \Omega$ on $\Omega$, where $r_{\bar{x}}$ and $w_{\bar{x}}$ are any integers such that $r_{\bar{x}}+w_{\bar{x}}=p$. In other words, we show that there exists a function $\eta: \Omega \times \Omega \rightarrow R^{n}$ such that 3.16 is fulfilled.

First, we consider the case when $\bar{x}$ is a vector Fritz John critical point of the problem (3.1). Hence, by assumption, $\bar{x} \in \Omega$ is a weakly $K$-efficient solution of the problem (3.1). Therefore, by Definition 3.2, the following relation

$$
f(x)-f(\bar{x}) \notin-\operatorname{int} K
$$

holds for all $x \in \Omega$. Then we set $r_{\bar{x}}$ and $w_{\bar{x}}$ as any integers such that $r_{\bar{x}}+w_{\bar{x}}=p$ and, moreover, $\eta$ is assumed to be any function $\eta: \Omega \times \Omega \rightarrow R^{n}$.

Now, we consider the case when $x$ and $\bar{x}$ are any feasible points for 3.1 such that $f(x)-f(\bar{x}) \in-\operatorname{int} K$. Therefore, by Definition $3.2, \bar{x}$ is not a weakly $K$-efficient solution in the problem (3.1). By assumption, we conclude from here that $\bar{x}$ is not a vector Fritz John critical point. Hence, by Definition 3.4, it follows that

$$
0_{n} \notin \bar{\lambda}^{T} \partial f(\bar{x})+\bar{\mu}^{T} \partial g(\bar{x})+\bar{\vartheta}^{T} \partial h(\bar{x}) .
$$


In other words, the relation

$$
\bar{\lambda}^{T} A+\bar{\mu}^{T} B+\bar{\vartheta}^{T} D \neq 0_{n}
$$

holds for all $A \in \partial f(\bar{x}), B \in \partial g(\bar{x}), D \in \partial h(\bar{x})$. Let us denote $\bar{\vartheta}=\left(\bar{\vartheta}^{r \bar{x}}, \bar{\vartheta}^{w_{\bar{x}}}\right) \in R^{p}$, where $\bar{\vartheta}^{r_{\bar{x}}}$ is an $r_{\bar{x}}=\left|T_{-}^{\bar{\vartheta}}\right|$-dimensional vector such that each its component is negative and $\bar{\vartheta}^{w_{\bar{x}}}$ is a $w_{\bar{x}}=\left|T_{+}^{\bar{\vartheta}}\right|$-dimensional vector such that is each its component is nonnegative. Further, let $h_{-}^{r_{\bar{x}}}=\left(h_{t}: t \in T_{-}^{\bar{\vartheta}}\right): R^{n} \rightarrow R^{r_{\bar{x}}}$ and $h_{+}^{w_{\bar{x}}}=\left(h_{t}: t \in T_{+}^{\bar{\vartheta}}\right): R^{n} \rightarrow R^{w_{\bar{x}}}$. Thus, the above relation can be re-written in the following form

$$
\bar{\lambda}^{T} A+\bar{\mu}^{T} B+\left(-\bar{\vartheta}^{r_{\bar{x}}}\right)^{T}\left(-D_{-}^{r_{\bar{x}}}\right)+\left(\bar{\vartheta}^{w_{\bar{x}}}\right)^{T} D_{+}^{w_{\bar{x}}} \neq 0_{n}
$$

and it holds for all $A \in \partial f(\bar{x}), B \in \partial g(\bar{x}), D_{-}^{r_{\bar{x}}} \in \partial h^{r_{\bar{x}}}(\bar{x}), D_{+}^{w_{\bar{x}}} \in \partial h^{w_{\bar{x}}}(\bar{x})$, where $D_{-}^{r_{\bar{x}}}$ and $D_{+}^{w_{\bar{x}}}$ are $r_{\bar{x}} \times n$ and $w_{\bar{x}} \times n$-dimensional matrices, respectively. Hence, by Theorem 2.10 . it follows that there exists $y_{\bar{x}} \in R^{n}$ such that

$$
\left[\begin{array}{c}
A \\
B \\
-D_{-}^{r_{\bar{x}}} \\
D_{+}^{w_{\bar{x}}}
\end{array}\right] y_{\bar{x}} \in\left(-\operatorname{int} K \times-\operatorname{int} C \times\left(-\operatorname{int} R_{+}^{r_{\bar{x}}}\right) \times\left(-\operatorname{int} R_{+}^{w_{\bar{x}}}\right)\right) .
$$

Thus, 3.21 yields, respectively,

$$
A y_{\bar{x}} \in-\operatorname{int} K, \quad B y_{\bar{x}} \in-\operatorname{int} C, \quad-D_{-}^{r_{\bar{x}}} y_{\bar{x}} \in-\operatorname{int} R_{+}^{r_{\bar{x}}}, \quad D_{+}^{w_{\bar{x}}} y_{\bar{x}} \in-\operatorname{int} R_{+}^{w_{\bar{x}}} .
$$

If we set $y_{\bar{x}}=\eta(x, \bar{x}) \in R^{n}$ in 3.22 , then relations (3.16) are fulfilled. Thus, we have shown that there exists $\eta: \Omega \times \Omega \rightarrow R^{n}$ such that relations 3.16 are fulfilled. Then, by Definition 3.10, the problem (3.1) is $\left(K \times C \times R_{+}^{w_{\bar{x}}} \times R_{+}^{r_{\bar{x}}}\right)-F J$-pseudoinvex at each $\bar{x} \in \Omega$ on $\Omega$, where $r_{\bar{x}}$ and $w_{\bar{x}}$ are any integers satisfying $r_{\bar{x}}+w_{\bar{x}}=p$. Thus, the problem (3.1) is $\left(K \times C \times R_{+}^{p}\right)$ - $F J$-pseudoinvex on $\Omega$ (see Remark 3.11 ).

Now, we present a class of nondifferentiable multiobjective programming problems in which every component of the involved functions is locally Lipschitz which are characterized by the property that each vector Kuhn-Tucker critical point is a $K$-efficient point in such vector optimization problems.

Theorem 3.21. Let $\bar{x} \in \Omega$ be a vector Kuhn-Tucker critical point with the Lagrange multipliers $\bar{\lambda} \in K^{+} \backslash\{0\}, \bar{\mu} \in C^{+}$and $\bar{\vartheta} \in R^{p}$ and let $T_{w}^{\bar{\vartheta}}=\left\{t \in\{1, \ldots, p\}: \bar{\vartheta}_{t} \geq\right.$ $0\}$ and $T_{r}^{\bar{\vartheta}}=\left\{t \in\{1, \ldots, p\}: \bar{\vartheta}_{t}<0\right\}$. Further, assume that the problem 3.1 is $\left(K \times C \times R_{+}^{r} \times R_{+}^{w}\right)$-KT-pseudoinvex at $\bar{x}$ on $\Omega$, where $r=\left|T_{-}^{\bar{\vartheta}}\right|$ and $w=\left|T_{+}^{\bar{\vartheta}}\right|$. Then $\bar{x}$ is a K-efficient solution of the problem (3.1). 
Proof. Assume that the problem (3.1) is $\left(K \times C \times R_{+}^{w} \times R_{+}^{r}\right)-K T$-pseudoinvex at the point $\bar{x} \in \Omega$ on $\Omega$ with respect to the same function $\eta$, where $w=\left|T_{+}^{\bar{\vartheta}}\right|, r=\left|T_{-}^{\bar{\vartheta}}\right|$. Further, assume that $\bar{x}$ is a vector Kuhn-Tucker critical point with the Lagrange multipliers $\bar{\lambda} \in K^{+} \backslash\{0\}$, $\bar{\mu} \in C^{+}$and $\bar{\vartheta} \in R^{p}$ in the problem (3.1). We show that $\bar{x}$ is a $K$-efficient solution of the problem (3.1). Suppose, contrary to the result, that $\bar{x}$ is not a $K$-efficient solution of the problem (3.1). Then, by Definition 3.3, there exists $\widetilde{x} \in \Omega$ such that

$$
f(\widetilde{x})-f(\bar{x}) \in-K \backslash\{0\} .
$$

By $\bar{x} \in \Omega$ and $\widetilde{x} \in \Omega$, we have

$$
-g(\widetilde{x}) \in C, \quad-g(\bar{x}) \in C, \quad h(\bar{x})=0 .
$$

Hence, by Definition 3.14, 3.23) and (3.24) yield that the relations

$$
\begin{gathered}
A \eta(\widetilde{x}, \bar{x}) \in-\operatorname{int} K, \\
B \eta(\widetilde{x}, \bar{x}) \in-\operatorname{int} C, \\
-D_{t} \eta(\widetilde{x}, \bar{x}) \in-\operatorname{int} R_{+}, \quad \forall t \in T_{-}^{\bar{\vartheta}}, \\
D_{t} \eta(\widetilde{x}, \bar{x}) \in-\operatorname{int} R_{+}, \quad \forall t \in T_{+}^{\bar{\vartheta}}
\end{gathered}
$$

hold for any $A \in \partial f(\bar{x}), B \in \partial g(\bar{x}), D_{t} \in \partial h_{t}(\bar{x}), t \in T_{-}^{\bar{\vartheta}} \cup T_{+}^{\bar{\vartheta}}$, respectively. Since $\bar{\lambda} \in K^{+} \backslash\{0\}$ and $\bar{\mu} \in C^{+}$, by Lemma 2.6, (3.25) and (3.26) yield

$$
\bar{\lambda}^{T} A \eta(\widetilde{x}, \bar{x})+\bar{\mu}^{T} B \eta(\widetilde{x}, \bar{x})<0 .
$$

Thus, 3.27) and (3.28) imply, respectively,

$$
\begin{aligned}
& \bar{\vartheta}_{t}^{T} D_{t} \eta(\widetilde{x}, \bar{x}) \leq 0, \quad \forall t \in T_{-}^{\bar{\vartheta}}, \\
& \bar{\vartheta}_{t}^{T} D_{t} \eta(\widetilde{x}, \bar{x}) \leq 0, \quad \forall t \in T_{+}^{\bar{\vartheta}} .
\end{aligned}
$$

Adding both sides of $3.29-3.31$, we obtain that the inequality

$$
\left[\bar{\lambda}^{T} A+\bar{\mu}^{T} B+\bar{\vartheta}^{T} D\right] \eta(\widetilde{x}, \bar{x})<0
$$

holds for any $A \in \partial f(\bar{x}), B \in \partial g(\bar{x}), D \in \partial h(\bar{x})$, which contradicts the assumption that $\bar{x}$ is a vector Kuhn-Tucker critical point $\bar{x}$ (with Lagrange multipliers $\bar{\lambda} \in K^{+} \backslash\{0\}$, $\bar{\mu} \in C^{+}$and $\bar{\vartheta} \in R^{p}$ ). This means that $\bar{x}$ is a $K$-efficient solution of the problem (3.1) and completes the proof of this theorem.

Theorem 3.22. Let every vector Karush-Kuhn-Tucker critical point of the considered multiobjective programming problem (3.1) be its K-efficient solution. Then, the problem (3.1) is $\left(K \times C \times R_{+}^{r_{\bar{x}}} \times R_{+}^{w_{\bar{x}}}\right)-K T$-pseudoinvex at each point $\bar{x} \in \Omega$ on $\Omega$, where $r_{\bar{x}}$ and $w_{\bar{x}}$ are any integers such that $r_{\bar{x}}+w_{\bar{x}}=p$. In other words, the problem (3.1) is $\left(K \times C \times R_{+}^{p}\right)$-KT-pseudoinvex on $\Omega$. 
Proof. Proof of this theorem is similar to the proof of Theorem 3.20 .

Now, we present an example of a nonconvex nondifferentiable multiobjective programming problem to illustrate the results established in the paper.

Example 3.23. Consider the following nonconvex nondifferentiable vector optimization problem

$$
K \text {-minimize } f(x) \text { subject to }-g(x) \in C, h(x)=\{0\}_{R},
$$

where $f=\left(f_{1}, f_{2}\right): R^{2} \rightarrow R^{2}, g=\left(g_{1}, g_{2}\right): R^{2} \rightarrow R^{2}, h: R^{2} \rightarrow R$ are defined as follows:

$$
\begin{aligned}
& f_{1}\left(x_{1}, x_{2}\right)= \begin{cases}-2 x_{1}^{2}-2 x_{2}^{2}-\frac{1}{2} x_{1}-\frac{1}{2} x_{2}-1 & \text { if } x_{1} \geq 0, x_{2} \geq 0, \\
-x_{1}^{2}-x_{2}^{2}-1 & \text { otherwise, }\end{cases} \\
& f_{2}\left(x_{1}, x_{2}\right)= \begin{cases}x_{1}^{2}+x_{2}^{2}-2 x_{1}-2 x_{2}+1 & \text { if } x_{1} \geq 0, x_{2} \geq 0, \\
x_{1}^{2}+x_{2}^{2}-x_{1}-x_{2}+1 & \text { otherwise, }\end{cases} \\
& g_{1}\left(x_{1}, x_{2}\right)= \begin{cases}-x_{1}^{2}-x_{2}^{2}-\frac{1}{4} x_{1}-\frac{1}{4} x_{2} & \text { if } x_{1} \geq 0, x_{2} \geq 0, \\
-x_{1}^{2}-x_{2}^{2} & \text { otherwise, }\end{cases} \\
& g_{2}\left(x_{1}, x_{2}\right)= \begin{cases}-2 x_{1}^{2}-2 x_{2}^{2}-\frac{3}{2} x_{1}-\frac{3}{2} x_{2} & \text { if } x_{1} \geq 0, x_{2} \geq 0, \\
-x_{1}^{2}-x_{2}^{2}-x_{1}-x_{2} & \text { otherwise, }\end{cases} \\
& h_{1}\left(x_{1}, x_{2}\right)= \\
& \left|x_{1}\right|-\left|x_{2}\right| .
\end{aligned}
$$

Further, $K$ and $C$ are closed convex cones with nonempty interiors in $R^{2}$ defined as follows:

$$
K=\left\{(x, y) \in R^{2}: y \geq x \geq 0\right\}, \quad C=\left\{(x, y) \in R^{2}: y \geq x \geq 0\right\}
$$

Then, the set of all feasible solutions $\Omega=\left\{\left(x_{1}, x_{2}\right) \in R^{2}:\left[\left(x_{1} \geq 0 \wedge x_{2} \geq 0\right) \vee\left(x_{1}+x_{2} \geq\right.\right.\right.$ $\left.0)] \wedge\left|x_{1}\right|-\left|x_{2}\right|=0\right\}$ and $\bar{x}=\left(\bar{x}_{1}, \bar{x}_{2}\right)=(0,0) \in \Omega$. Further, by Definition 2.12, we have

$$
\begin{gathered}
\partial f_{1}(0,0)=\left[-\frac{1}{2}, 0\right] \times\left[-\frac{1}{2}, 0\right], \quad \partial f_{2}(0,0)=[-2,-1] \times[-2,-1] \\
\partial g_{1}(0,0)=\left[-\frac{1}{4}, 0\right] \times\left[-\frac{1}{4}, 0\right], \quad \partial g_{2}(0,0)=\left[-\frac{3}{2},-1\right] \times\left[-\frac{3}{2},-1\right], \\
\partial h_{1}(0,0)=[-1,1] \times[-1,1] .
\end{gathered}
$$

Note that $\bar{x}=\left(\bar{x}_{1}, \bar{x}_{2}\right)=(0,0)$ is a vector Kuhn-Tucker critical point in the considered nonconvex nondifferentiable vector optimization problem 3.32). Indeed, if we set

$$
\bar{\lambda}=(-1,1) \in K^{+}, \quad \bar{\mu}=(-1,1) \in C^{+}, \quad \bar{\vartheta}_{1}=2,
$$


then, by Definition 3.6, $\bar{x}=\left(\bar{x}_{1}, \bar{x}_{2}\right)=(0,0)$ is a vector Kuhn-Tucker critical point in the problem (3.32) (with Lagrange multipliers $\bar{\lambda} \in K^{+}, \bar{\mu} \in C^{+}$and $\bar{\vartheta} \in R$ given above). Further, let $\eta: \Omega \times \Omega \rightarrow R^{2}$ be defined by

$$
\eta(x, \bar{x})=\left[\begin{array}{l}
\bar{x}_{1}^{2}-x_{1}^{2} \\
\bar{x}_{2}^{2}-x_{2}^{2}
\end{array}\right]
$$

Then, it can be shown, by Definition 3.14 , that the problem 3.32 is $\left(K \times C \times R_{+}^{1}\right)$ $K T^{+}$-pseudoinvex at the point $\bar{x} \in \Omega$ on $\Omega$ with respect to $\eta$ given above. Thus, by Theorem 3.21 , it follows that $\bar{x}$ is a $K$-efficient solution of the considered nonconvex nondifferentiable vector optimization problem 3.32 .

\section{Conclusions}

In the paper, a nonsmooth multiobjective programming problem with both cone and equality constraints has been considered. Definitions of vector critical points in the sense of Fritz John and in the sense of Kuhn-Tucker have been introduced for such a nondifferentiable vector optimization problem. Subsequently, the relationships between these vector critical points and (weakly) $K$-efficient solutions in the considered nonsmooth multiobjective programming problem have been derived. Namely, the necessary and sufficient optimality conditions have been proved for critical points in the sense of Fritz John and in the sense of Kuhn-Tucker. In order to prove the necessary and sufficient optimality conditions for (weakly) $K$-efficiency by the help of vector critical points, new concepts of generalized convexity, namely cone- $F J$-pseudoinvexity and cone- $K T$-pseudoinvexity have been introduced for the considered nonsmooth vector optimization problem with both cone and equality constraints. Thus, the characterization of vector critical points have been extended to the largest class of nonconvex nodifferentiable vector optimization problems with both cone and equality constraints, in comparison to the similar results existing in the literature.

\section{References}

[1] T. Antczak, A new approach to multiobjective programming with a modified objective function, J. Global Optim. 27 (2003), no. 4, 485-495.

[2] _ Optimality and duality for nonsmooth multiobjective programming problems with V-r-invexity, J. Global Optim. 45 (2009), no. 2, 319-334. 
[3] _ Proper efficiency conditions and duality results for nonsmooth vector optimization in Banach spaces under $(\Phi, \rho)$-invexity, Nonlinear Anal. 75 (2012), no. 6, 3107-3121.

[4] M. Arana-Jiménez, A. Rufián-Lizana, R. Osuna-Gómez and G. Ruiz-Garzón, A characterization of pseudoinvexity in multiobjective programming, Math. Comput. Modelling 48 (2008), no. 11-12, 1719-1723.

[5] M. Arana-Jiménez, G. Ruiz-Garzón and A. Rufián-Lizana, Pseudoinvexity: A good condition for efficiency and weak efficiency in multiobjective mathematical programming, characterization, in: Optimality Conditions in Vector Optimization, 1-16, Bentham Science, Sharjah, 2012.

[6] D. Bhatia and P. Jain, Generalized $(F, \rho)$-convexity and duality for non smooth multiobjective programs, Optimization 31 (1994), no. 2, 239-244.

[7] N. Bourbaki, Topological Vector Spaces, Springer-Verlag, Berlin, 2003.

[8] S. Boyd and L. Vandenberghe, Convex Optimization, Cambridge University Press, Cambridge, 2004.

[9] J. W. Chen, Y. J. Cho, J. K. Kim and J. Li, Multiobjective optimization problems with modified objective functions and cone constraints and applications, J. Global Optim. 49 (2011), no. 1, 137-147.

[10] J. W. Chen, Z. P. Wan and Y. Zheng, Optimality conditions and duality for nonsmooth multiobjective optimization problems with cone constraints and applications, http://www.optimization-online.org/DBFILE/2010/07/2672.pdf

[11] F. H. Clarke, Generalized gradients and applications, Trans. Amer. Math. Soc. 205 (1975), 247-262.

[12] _ Optimization and Nonsmooth Analysis, Canadian Mathematical Society Series of Monographs and Advanced Texts, A Wiley-Interscience Publication, John Wiley \& Sons, New York, 1983.

[13] B. D. Craven, Nondifferentiable optimization by smooth approximations, Optimization 17 (1986), no. 1, 3-17.

[14] _ Nonsmooth multiobjective programming, Numer. Funct. Anal. Optim. 10 (1989), no. 1-2, 49-64.

[15] B. D. Craven and X. Q. Yang, A nonsmooth version of alternative theorem and nonsmooth multiobjective programming, Utilitas Math. 40 (1991), 117-128. 
[16] J. Dattorro, Convex Optimization and Euclidean Distance Geometry, Meboo, California, 2015, version 2011.04.25.

[17] N. Dunford and J. T. Schwartz, Linear Operators I: General Theory, Pure and Applied Mathematics 7, Interscience Publishers, New York, 1958.

[18] I. Ekeland, On the variational principle, J. Math. Anal. Appl. 47 (1974), 324-353.

[19] G. Giorgi and A. Guerraggio, The notion of invexity in vector optimization: Smooth and nonsmooth case, in: Generalized Convexity, Generalized Monotonicity: Recent Results, (Luminy, 1996), 389-405, Nonconvex Optim. Appl. 27, Kluwer Academic Publishers, Dordrecht, 1998.

[20] C. Gutiérrez, B. Jiménez, V. Novo and G. Ruiz-Garzón, Vector critical points and efficiency in vector optimization with Lipschitz functions, Optim. Lett. 10 (2016), no. $1,47-62$.

[21] V. I. Ivanov, On the optimality of some classes of invex problems, Optim. Lett. 6 (2012), no. 1, 43-54.

[22] M. H. Kim and G. M. Lee, On duality theorems for nonsmooth Lipschitz optimization problems, J. Optim. Theory Appl. 110 (2001), no. 3, 669-675.

[23] D. S. Kim and S. Schaible, Optimality and duality for invex nonsmooth multiobjective programming problems, Optimization 53 (2004), no. 2, 165-176.

[24] G. M. Lee, Nonsmooth invexity in multiobjective programming, J. Inform. Optim. Sci. 15 (1994), no. 1, 127-136.

[25] L. Li and J. Li, Equivalence and existence of weak Pareto optima for multiobjective optimization problems with cone constraints, Appl. Math. Lett. 21 (2008), no. 6, 599-606.

[26] M. Minami, Weak Pareto-optimal necessary conditions in a nondifferentiable multiobjective program on a Banach space, J. Optim. Theory Appl. 41 (1983), no. 3, $451-461$.

[27] S. K. Mishra, S. Wang and K. K. Lai, V-invex Functions and Vector Optimization, Springer Optimization and its Applications 14, Springer, New York, 2008.

[28] _ Generalized Convexity and Vector Optimization, Nonconvex Optimization and its Applications 90, Springer-Verlag, Berlin, 2009. 
[29] R. Osuna-Gómez, A. Rufián-Lizana and P. Ruíz-Canales, Invex functions and generalized convexity in multiobjective programming, J. Optim. Theory Appl. 98 (1998), no. 3, 651-661.

[30] A. L. Peressini, Ordered Topological Vector Spaces, Harper \& Row, New York, 1967.

[31] T. W. Reiland, Generalized invexity for nonsmooth vector-valued mappings, Numer. Funct. Anal. Optim. 10 (1989), no. 11-12, 1191-1202.

[32] R. T. Rockafellar, Convex Analysis, Princeton Landmarks in Mathematics, Princeton Paperbacks, Princeton University Press, Princeton, NJ, 1997.

[33] W. Rudin, Functional Analysis, McGraw-Hill Series in Higher Mathematics, McGrawHill Book Company, New York, 1973.

[34] M. Soleimani-damaneh, On optimality and duality for multiple-objective optimization under generalized type I univexity, Int. J. Comput. Math. 86 (2009), no. 8, 1345-1354.

[35] S. K. Suneja, S. Khurana and M. Bhatia, Optimality and duality in vector optimization involving generalized type I functions over cones, J. Global Optim. 49 (2011), no. 1, 23-35.

[36] S. K. Suneja, S. Khurana and Vani, Generalized nonsmooth invexity over cones in vector optimization, European J. Oper. Res. 186 (2008), no. 1, 28-40.

[37] N. L. Tung and D. V. Luu, Optimality conditions for nonsmooth multiobjective optimization problems with general inequality constraints, J. Nonlinear Funct. Anal. 2018 (2018), Article ID 2, 1-15.

[38] N. D. Yen and P. H. Sach, On locally Lipschitz vector-valued invex functions, Bull. Austral. Math. Soc. 47 (1993), no. 2, 259-272.

Tadeusz Antczak and Marcin Studniarski

Faculty of Mathematics and Computer Science, University of Łódź Banacha 22, 90-238

Łódź, Poland

E-mail address: tadeusz.antczak@wmii.uni.lodz.pl,

marcin.studniarski@wmii.uni.lodz.pl 